\title{
VARIATION IN DURATION OF HOSPITAL STAY BETWEEN HOSPITALS AND BETWEEN DOCTORS WITHIN HOSPITALS
}

\author{
Gert P. Westert, ${ }^{1}$ Anna P. Nieboer ${ }^{2}$ and Peter P. Groenewegen ${ }^{3}$ \\ ${ }^{1}$ University of Twente, Department of Sociology (BSK), P.O. Box 217, 7500 AE Enschede, The \\ Netherlands, ${ }^{2}$ University of Groningen, Interuniversity Center for Sociology, Groningen, The Nether- \\ lands and ${ }^{3}$ Netherlands Institute of Primary Health Care, and University of Utrecht, Department of \\ Human Geography and Department of Sociology, Utrecht, The Netherlands
}

\begin{abstract}
Whether one examines the average length of hospital stay at the level of geographic areas, at the level of hospitals, or at the level of doctors, length-of-stay figures are known to vary widely. Even for hospital admissions for comparable surgical procedures among comparable groups of patients, significant length-of-stay variations have been reported. As is the case for variations in the occurrence of common surgical procedures, the overall conclusion is that large variations in duration of hospital stay associated with these common surgical procedures are the rule rather than the exception. The objective of the study is to examine whether variations in hospital medical practice, indicated by the duration of hospital stay in this study, can be reduced to differences in practice style between individual doctors within the same institutional setting or to differences in practice style between groups of doctors within the same institutional setting. The latter is assumed to be the combined effect of restrictions on the (hospital) supply side and the predilection of doctors to conform to the practice of immediate colleagues. It was found out that the variation in length of hospital stay, adjusted for patient case-mix, within hospitals is much smaller than the length-of-stay variation between different hospitals. The within hospital variation between (partnership of) doctors is in most of the cases statistically insignificant. Doctors working in more than one hospital on average choose a length of stay close to the average length of stay prevailing in the different hospitals.
\end{abstract}

Key words-specialist care, length of hospital stay, surgical procedures, The Netherlands

\section{INTRODUCTION}

Whether one examines the average length of hospital stay at the level of geographic areas, at the level of hospitals, or at the level of doctors, length-of-stay figures are known to vary widely [1-3]. Even for hospital admissions for comparable surgical procedures among comparable groups of patients, significant length-of-stay variations have been reported [4-6]. As is the case for variations in the occurrence of common surgical procedures, the overall conclusion is that large variations in duration of hospital stay associated with these common surgical procedures are the rule rather than the exception [6].

The explanation of these variations in the recent literature draws heavily on the variation in practice style concept, introduced by Wennberg and various collaborators $[6,7]$. From this concept two ideas have been developed by these authors. First, the idea of a surgical signature, which states that individual doctors all have their own individual work habits and have their own ideas about what is good medical practice. Second, the idea of professional uncertainty, which is that individual doctors differ in medical practice because they differ in the degree of perceived uncertainty regarding the use and management of different surgical procedures. Both notions imply that variation in hospital outcome measures is mainly found on the individual doctor's level.
The question to be answered in this article is whether the differences in length of hospital stay for common surgical procedures are determined by individual differences between doctors in practice style or by systematic differences related to the work (hospital) setting. If the explanatory concept of individual practice style holds true, the largest variation must be found between individual doctors, irrespective of their work setting. An alternative hypothesis is that variations between doctors are related to systematic differences in work settings. First, this implies that variation between doctors working in the same hospital setting is relatively small, compared to the variation between hospitals. Second, this implies that doctors working in more than one hospital tend to conform to the usual practice in the different hospitals.

Behind this alternative hypothesis lies two arguments. First, because immediate colleagues are exposed to the same institutional (hospital) restrictions they probably will be induced to make similar lengthof-stay decisions. These restrictions concern e.g. the bed capacity of the hospital, the level of technical facilities and hospital management policies. Second, the hospital work setting is also a social setting which exerts pressure towards conformity. Individual doctors tend to minimize the probability of being criticized by immediate colleagues with respect to 
their medical decision making. To achieve this, the appropriate maxim is 'safety in numbers'. As Eddy puts it: "If it is admitted that the uncertainty surrounding the use of a procedure is great, and that there is no way to identify for certain what is best, or prove that any particular action is right or wrong, the safest and most comfortable position is to do what others are doing. A physician who follows the practices of his or her colleagues is safe from criticism, free from having to explain his or her actions, and defended by the concurrence of colleagues" [8, p. 86]. Although Eddy alludes to decisions concerning the appropriateness of surgical procedures, we think that the maxim can also be applied to length-of-stay decisions. With respect to length-of-stay decisions 'safety in numbers' means that: immediate colleagues on the same medical ward tend to choose, on average, the same length of stay for the same surgical procedures.

We expect that the largest and most important variations in duration of hospital stay will not be found on the level of the individual doctor but on the level of groups of doctors. In the literature we found only one empirical study which addresses the same problem. This study fully supports our hypotheses [9]. However, the conclusions which can be drawn from this study are limited. First, because only one surgical procedure was involved in the study: inguinal hernia repair. Second, although some cases were excluded from the analysis the average postoperative length of stay was not adjusted for patient case-mix variables, and therefore the results might be biased to some extent. Griffiths et al, do report that age has no significant effect on length of hospital stay [9].

Mainly because of these two points it is worthwhile to repeat the empirical test of our hypotheses by using case-mix adjusted length-of-stay data for a number of different surgical procedures. Repeated empirical testing is also suitable because our data are more recent $(1982,1986$ and 1987 vs 1970). The data were collected in a different health care system (Dutch health care system vs the British National Health Service).

We will test the following two hypotheses:

- hypothesis 1: the variation in procedure-specific length of stay within hospitals between the (partnerships of) doctors is insignificant, compared to the variation in procedure-specific length of stay between hospitals.

- hypothesis 2: if a doctor works in two or more hospitals with different average procedure-specific length of hospital stays, then the chosen average procedure-specific length of stay by this doctor varies between the hospitals, in the direction of the average stay in the hospital where he or she performs the specific procedure.

\section{DATA AND METHODS}

Two databases were used to test the hypotheses. The first database (A) is nationwide and contains the insurance-records of all publicly insured patients (about $65 \%$ of the Dutch population). For our study we selected patients who were admitted to a hospital in 1982 or 1986 for appendectomy, gall bladder extirpation, nasal septum correction or meniscus extirpation.

The information in the database is derived from the treatment-specific claims of fee for services paid to doctors. On every patient record the doctor's identificationcode is available, but since many doctors practices are partnerships, and use only one code for the practice, the lowest level available is in many cases not the level of the individual doctor, but of the partnership. Because the purpose of our study is to analyze between-doctor variation within hospitals, hospitals with only one doctor or partnershipcode were excluded from the analysis in testing the first hypothesis. In Table 1, columns 2 and 3, the number of hospitals and doctors are shown. In most of the remaining cases the doctor is the unit of analysis and not the partnership.

Patients with an extremely long duration of stay were excluded from database $A$ as well. The reasons for this is to prevent distortion of the average duration of stay at the level of doctors and hospitals. The definition of extremely long is the procedure-specific average duration of hospital stay plus two times the standard deviation.

Additionally, only doctors (or partnerships) who performed the surgical procedure at least 10 times in the year of discharge were accepted. This selection was carried out, for statistical reasons, to prevent instability of the doctor's chosen average length of stay. The lower the number of observations per doctors the lower the reliability of the doctor's (procedure-specific) length-of-stay practice.

Table 1. Number of hospitals (2), number of different (partnerships of) doctors working within these hospitals (3) and results of the one-way analysis of variance between and within hospitals $(4,5)$ for the selected surgical procedures (1)

\begin{tabular}{|c|c|c|c|c|}
\hline $\begin{array}{l}\text { l } \\
\text { Surgical } \\
\text { procedure }\end{array}$ & $\begin{array}{c}2 \\
n \\
\text { hospitals }\end{array}$ & $\begin{array}{c}3 \\
n \\
\text { doctors }\end{array}$ & $\begin{array}{c}4 \\
F^{2}\end{array}$ & $\begin{array}{l}5 \\
P\end{array}$ \\
\hline \multicolumn{5}{|l|}{1982} \\
\hline Appendectomy & 26 & 61 & 2.1 & $(0.02)$ \\
\hline Gall bladder extirpation & 20 & 43 & 3.2 & $(<0.01)$ \\
\hline Meniscus extirpation $(1)^{b}$ & 4 & 8 & 12.1 & $(0.02)$ \\
\hline Meniscus extirpation $(2)^{b}$ & 10 & 21 & 2.1 & $(0.12)$ \\
\hline Nasal septum operation & 16 & 36 & 5.4 & $(<0.01)$ \\
\hline \multicolumn{5}{|l|}{1986} \\
\hline Appendectomy & 45 & 116 & 2.7 & $(<0.01)$ \\
\hline Gall bladder extirpation & 27 & 59 & 3.3 & $(<0.01)$ \\
\hline Meniscus extirpation $(1)^{b}$ & 2 & 4 & 57.5 & $(0.02)$ \\
\hline Meniscus extirpation (2) & 37 & 86 & 13.1 & $(<0.01)$ \\
\hline Nasal septum operation & 39 & 96 & 7.9 & $(<0.01)$ \\
\hline
\end{tabular}

"If the $F$-statistic significantly differs from 1 , the between hospital variability exceeds the variability within hospitals.

b (1) Stands for general surgeons and (2) stands for orthopaedic surgeons performing the meniscus extirpation. 
The percentage of patients left in the database, and used to test both hypothesis 1 and 2, varied from $19 \%$ for the gall bladder extirpation in 1982 to $54 \%$ for the meniscus extirpation in 1986.

Because it cannot be ascertained whether the information in the nationwide database (A) was based on average length of stay linked to a partnership or linked to an individual doctor, an additional, second database was used for the analysis.

Database (B) contains length-of-stay data of patients who underwent either an appendectomy $(N=642)$ or an inguinal hernia repair $(N=727)$ in 1987 in five general hospitals. A few patients were excluded from the analysis because their length of stay was extremely long. Two doctors were excluded from the analysis as well because they treated fewer than 10 patients. This left 621 patients who underwent an appendectomy and 709 patients who underwent inguinal hernia repair. Twenty-three doctors working in five general hospitals (all located in one Dutch province) were included in the analysis, four of whom operated at more than one hospital.

The length of patient's hospital stay is adjusted for case-mix by regressing available indicators for the health status of the patient and the complexity of the case on the length of stay. The length of stay in database $A$ was adjusted for age, sex, number of additional procedures, number of medical specialties involved, and repetition of the main surgical procedure during the same hospital admission on another day. The length of stay in database $B$ was adjusted for: age, sex, emergency operation, the number of additional medical diagnoses, and the number of different surgical procedures involved.

For both databases an adjusted procedure-specific length of hospital stay score was computed for every patient as follows. First, the residuals from the regression analysis with the statistically significant case-mix variables as independent variables were computed. Second, the residuals and the average procedure-specific length of stay were added. This 'adjusted length-of-stay score' was aggregated to the level of the doctor (or partncrship).

For both databases the same methods of statistical analysis were used to test the two hypotheses. The first hypothesis was tested with oneway analysis of variance with one factor, the hospital. Variability in aggregated length of stay is divided into two components: one attributable to differences between hospitals, and one attributable to variability between doctors within hospitals. If the test statistic $F$ is significantly large, the between-hospital variability exceeds the variability within hospitals. Consequently, we conclude that doctors within hospitals tend to choose uniformly on length of stay.

The statistical analysis of hypothesis 2 is divided into two parts. In the first step the average length of hospital stay of the doctors working in different hospitals was calculated and pairwise $t$-tests were carried out. The pairwise $t$-test allows us to compare sample means and test their differences against expected values at any given level of probability. The $t$-tests were carried out only in cases where there was a significant difference in average length of stay in the hospitals in which the doctor worked.

In the second step we plotted the doctor's deviation in average length of stay between a hospital pair (vertical axis) against the length-of-stay deviation of the doctor's immediate colleagues for the same hospital pair (horizontal axis). If a doctor chooses a procedure-specific length of stay close to the hospital average where $s$ /he performs the operation (horizontal axis) then the doctor's deviation in length of stay between a hospital pair (vertical axis) lies on the expected (0-4) line (Fig. 2).

\section{RESULTS}

Table 1 gives the results of the analysis of variance for database $A$. In all but one row the value of the statistic $F$ differed significantly. The between-hospital variance in length of stay exceeds the within hospital variation. Only in the case of the meniscus extirpation (1982) performed by orthopaedic surgeons was the statistical analysis insignificant $(F=2.1, P=0.12)$. In this case more variance in length of stay was found within hospitals between doctors (or partnerships) than between hospitals. A closer look at the data of the meniscus extirpation (1982) revealed that one doctor's average length of stay strongly deviated from his/her colleagues in the same hospital. Excluding this outlier from the analysis increased the $F$-value to 5.60 $(P<0.01)$.

From database B, Fig. 1 shows the aggregated length-of-stay data disaggregated by the five hospitals where the operations were performed. The ten vertical lines show the length-of-stay variation within the five hospitals between individual doctors. In the case of appendectomy (first five vertical lines) the average length of stay between hospitals of the case-mix adjusted data varied from 6.8 to 8.9 days $(N=5)$, and between doctors from 6.5 to $9.5(N=23)$. For the inguinal herniorrhaphy the average length of stay between hospitals varied from 5.9 to $6.7(N=5)$, and between doctors from 3.9 to $7.1(N=23)$.

The analysis of variance to test length-of-stay differences between hospitals for appendectomy produced an $F$-value of $6.38(P<0.01)$. In terms of our hypothesis 1 this means that the variation in procedure-specific length of stay (between doctors) within hospitals is significantly smaller than between hospitals.

In the case of the inguinal herniorrhaphy no significant differences were found in average length of stay between hospitals. Because in the present study both the between-hospital variation and the within-hospital variation in length of stay in the latter case were close to zero, the test of the first hypothesis for these cases (inguinal herniorrhaphy) does not make sense. This does not mean that in these cases the hypothesis 


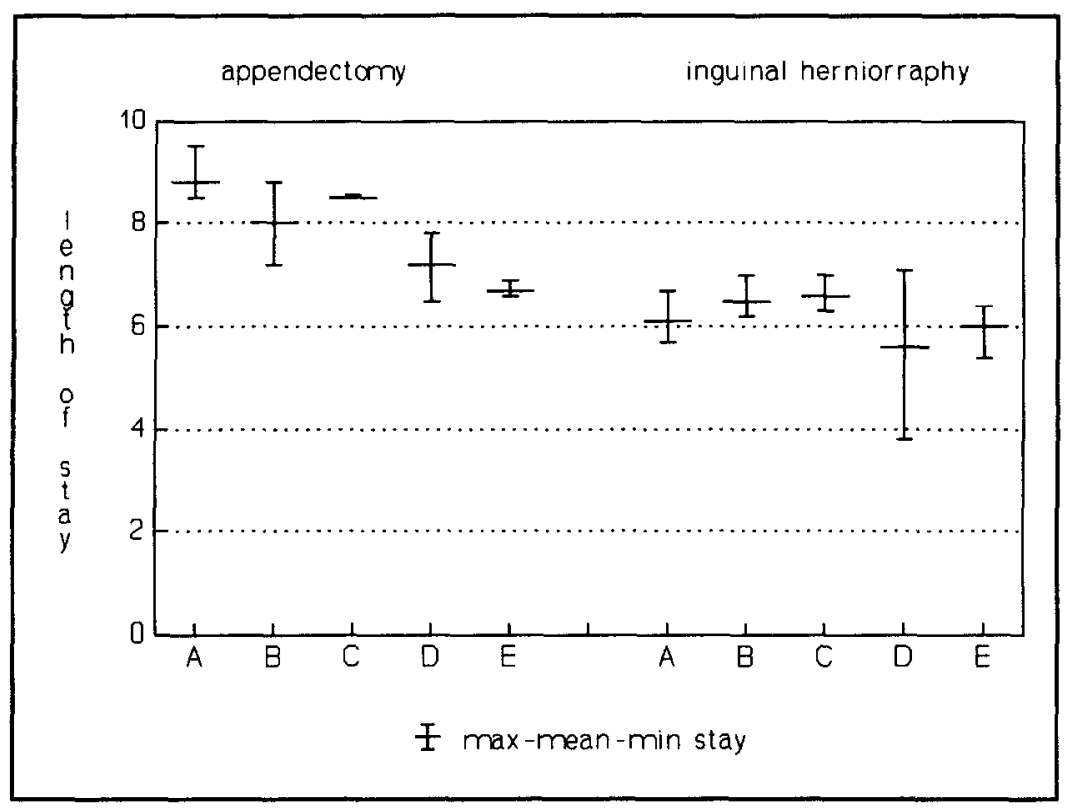

Fig. 1. Differences in length of stay between doctors within hospitals (database B).

is rejected. If the between-hospital variation had been close to zero and the within hospital variance signifcantly different from zero, then our hypothesis should have been refuted. In hospital D the latter seems the case, but it should be noted that hospital $D$ is a special case. This hospital is really three different hospitals, that recently merged. Following the merger in 1987 dispersed to different hospital locations; they did not work together in one single building. Part of the within-hospital variation, in the case of inguinal hernia repair, can therefore be explained by this organizational deviation. From the analysis of data- base B we conclude that both in the case of appendectomy and in the case of repair of inguinal hernia the doctors did conform to usual practice.

The results for the second hypothesis are shown in Table 2. Again two statistical procedures were used. First, from database $A$ the variance in average length of stay for doctors performing surgical procedures at different hospitals is shown. The first three columns are similar to those in Table 1. The fourth column shows the number of hospital pairs that could be compared. The fifth column shows the number of cases in which the average length of stay between

Table 2. Number of hospitals (2), number of doctors (3), number of hospital pairs (4), number of hospital pairs, of which the two hospitals differ statistically significant in length of stay (5), number of hospital pairs in the fifth column with intra-doctor variability (6)

\begin{tabular}{|c|c|c|c|c|c|}
\hline $\begin{array}{l}\quad 1 \\
\text { Surgical } \\
\text { Procedure }\end{array}$ & $\begin{array}{c}2 \\
\text { Number of } \\
\text { hospitals }\end{array}$ & $\begin{array}{c}3 \\
\text { Number of } \\
\text { doctors }\end{array}$ & $\begin{array}{c}4 \\
\text { Number of } \\
\text { hospital } \\
\text { pairs }\end{array}$ & $\begin{array}{c}5 \\
\text { Number of } \\
\text { hospital } \\
\text { pairs with } \\
\text { different } \\
\text { lengths of } \\
\text { stay }\end{array}$ & $\begin{array}{c}6 \\
\text { Number of } \\
\text { hospital } \\
\text { pairs with } \\
\text { intra-doctor } \\
\text { variability }\end{array}$ \\
\hline $\begin{array}{l}1982 \\
\text { Appendectomy } \\
\text { Gall bladder extirpation } \\
\text { Meniscus extirpation }{ }^{b} \\
\text { Nasal septum operation }\end{array}$ & $\begin{array}{r}5 \\
7 \\
55 \\
28\end{array}$ & $\begin{array}{r}2 \\
3 \\
25 \\
13\end{array}$ & $\begin{array}{r}4 \\
5 \\
35 \\
17\end{array}$ & $\begin{array}{c}0(0 \%) \\
3(60 \%) \\
17(49 \%) \\
9(53 \%)\end{array}$ & $\begin{array}{c}-\overline{-} \\
3(100 \%) \\
17(100 \%) \\
8(89 \%)\end{array}$ \\
\hline $\begin{array}{l}\text { Total } 1982 \\
1986 \\
\text { Appendectomy } \\
\text { Gall bladder extirpation } \\
\text { Meniscus extirpation } \\
\text { Nasal septum operation }\end{array}$ & $\begin{array}{r}95 \\
2 \\
2 \\
37 \\
20\end{array}$ & $\begin{array}{r}43 \\
1 \\
1 \\
21 \\
10\end{array}$ & $\begin{array}{r}1 \\
1 \\
21 \\
10\end{array}$ & $\begin{array}{c}29(48 \%) \\
1(100 \%) \\
0(0 \%) \\
13(62 \%) \\
4(40 \%)\end{array}$ & $\begin{array}{c}28(97 \%) \\
1(100 \%) \\
- \\
10(77 \%) \\
2(50 \%)\end{array}$ \\
\hline Total 1986 & 61 & 33 & 33 & $18(55 \%)$ & $13(72 \%)$ \\
\hline
\end{tabular}

Whenever a doctor works in 3 or more hospitals $(n), n(n-1) / 2$ pairwise tests were carried out.

- Means no difference in average length of stay between the hospitals so no $t$-test is carried out to test whether the doctor has different mean length of stay in the different hospitals.

${ }^{b}$ In contrast to Table 1 the meniscus extirpation is carried out in all suitable cases by orthopaedic surgeons. 


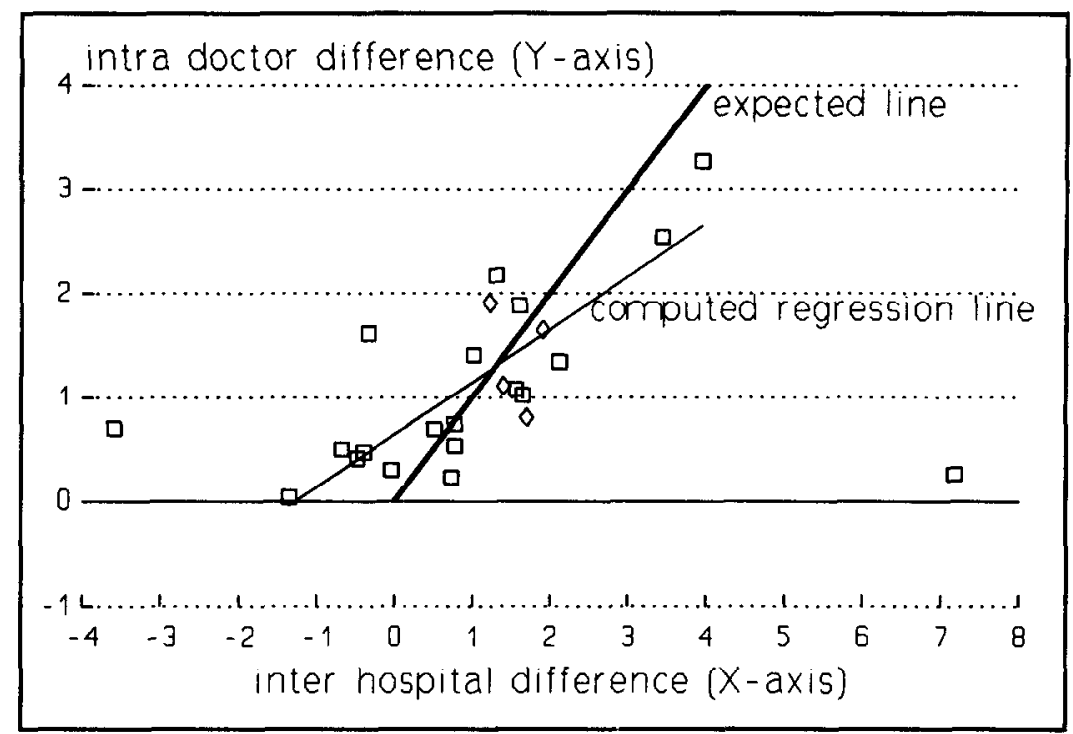

Fig. 2. Differences in length of stay per doctor in two hospitals (Y) compared to the difference in length of stay of his/her colleagues in the same hospital (X).

hospitals differed significantly from zero $(P<0.05)$, and between brackets the absolute figure in column 5 is divided by column 4 to show the percentage of suitable hospital pairs. Finally, the sixth column shows the number of times a doctor's (or medical partnership's) average length of hospital stay differed significantly in the different hospitals where $s /$ he was working, and between brackets the absolute figure in column 6 is divided by column 5 to show the percentage of hospital pairs with significant intra-doctor variation.

Table 2 supports the second hypothesis. Doctors working in different hospitals with different average procedure-specific length of stay make different medical decisions. This means that in those cases where the average length of stay in hospitals differed, the doctor's average length of stay in most cases ( 28 out of 29 in 1982 and 13 out of 18 in 1986) differed as well.

Because of the insignificant length-of-stay variation in the case of inguinal herniorrhaphy these cases were rejected from database $B$, and only surgical repair of appendicitis cases were analyzed. Four doctors were traced who performed appendectomies and worked in more than one hospital. One doctor worked in hospital B, C and E, one worked in A and B, and two doctors worked in hospital $B$ and $E$ (Fig. 1). Because the hospital averages of hospital A and B were approximately the same, only the length of stay decisions of the doctors working in the hospitalpairs $B$ and $E$ and $C$ and $E$ were compared. Consequently one doctor was excluded from the analysis. The $t$-tests showed statistically significant differences in decision-making in three out of four cases $(P<0.05)$.
The doctor's length-of-stay decision with the decision of his colleagues in the different hospitals where $s /$ he was working is compared, using linear regression (Fig. 2). On the vertical axis the absolute difference in length of stay of doctor $\mathrm{X}$ between hospital pair $i$ and $j$ is shown (intra-doctor difference) and on the horizontal axis the difference in length of stay of X's immediate colleagues in hospital $i$ and $j$ is shown (inter-hospital difference). For every possible pair of hospitals, $i$ and $j$, hospital $i$ was always the hospital with the longest duration of stay. Consequently the vertical axis has no negative values. To compute the value on the horizontal axis hospital $i$ and $j$ were put in the same sequence as on the vertical axis. For this figure the data from both databases were used. The doctors from database A are marked by an open square. The four doctors from database $B$ are marked by a diamond. Of the two outliers from database $\mathrm{A}$, one has a lower average length of stay in the hospital where his colleagues have the highest average length of stay. The other outlier hardly differs in his average length-of-stay decision despite the fact that the average length of hospital stay of his colleagues differs by more than seven days between the two hospitals. Apart from those two outliers, the chosen procedure-specific average length of stay of the doctor varies between the hospitals in the direction of the average stay in the hospital where $s / h e$ performed the specific procedure. A perfect prediction would find all coordinate points lying on the theoretically expected line. The regression line computed from all cases except the two outliers yields the expected positive tendency $(r=0.79, P=0.001)$. This demonstrates that doctors in different hospitals tend to choose a length of stay close to the usual practice in the hospital where the surgeries were performed. 


\section{DISCUSSION}

The purpose of our study was to find out whether the hospital level is a more suitable explanatory level to tackle the problem of length-of-stay variations than the level of the individual doctor. We predicted that individual doctors tend to conform to the (procedure-specific) length-of-stay practice in the hospital where they perform surgical procedures. We argued that the combination of similar institutional restrictions and the doctor's predilection to conform to conventional practice within the same hospital, since the individual doctor tries to avoid being criticized by colleagues, leads to small and insignificant length-of-stay variations between doctors within hospitals.

The results from empirical tests, on two different sets of hospital data, support our expectations. Additional support for our findings was found in the study of Griffiths et al., who carried out a study with a similar design, but in a health care system which is altogether different from the Dutch system [9]. There study was carried out in the late $1970 \mathrm{~s}$, using data from the British National Health Service. The authors report that for doctors operating in the same hospital, the average postoperative (in the case of inguinal hernia repair) stays were similar, whereas for doctors who operated at more than one hospital they were significantly different. In the study of Griffiths et al. length of stay was influenced more by the hospital to which the patient was admitted than by the doctor who managed the care of the patient.

Our study shows that the analytical level on which the observed variation can best be explained is the level of groups of doctors within hospitals. A plausible interpretation of these results is that doctors conform to local standards with regard to length-of-stay decisions. These standards that differ between hospitals or hospital wards. An important question for health policy is whether or not these local standards can be influenced.

First of all, we would like to draw attention to two policy developments which are relevant today in the Dutch health sector which will probably directly reduce the inter-local differences in length of stay standards considerably, independent of other interventions. The first development is the bed reduction policy which the Dutch government has pursued. When the number of beds is reduced in places where a surplus exists, the differences in length of stay will decrease. The availability of hospital beds is an important aspect of the institutional environment in which length of stay decisions are taken. Under the condition of scarcity of the number of available beds in a hospital the local length of stay standard tends to decrease. When differences between hospitals in the number of available beds are reduced, and when possibilities of substitution towards outpatient care are the same, differences in local standards tend to diminish [10]. This line of reasoning needs further empirical tests in the changing Dutch institutional context.

Second, in the 1980s the budget formula used to determine the level of hospital budgets in Dutch general hospitals was changed. According to the new system, the level of the hospital budget is a function of the number of places for medical specialists in the hospital, the number of admissions or outpatient visits, and the number of paticnt days rcalized. In the new budget formula, the patient day has become more or less a balancing entry. In contrast to former years, when the costs of hospital treatment were completely covered by a patient day fee, a hospital can scarcely exert any influence at all on the level of its own budget by realizing more or less patient days. Hospitals still receive a reimbursement per patient day, but this is far less than the cost price. This means that utilization of the available hospital beds by individual doctors will be less rewarded by the hospital management and immediate colleagues.

Other parameters have become more important under the new financing system. In the short term, this should have a negative effect on the mean length of hospital stay in the Netherlands. It is striking in this context that the mean length of stay in general hospitals has fallen before 11 days since 1989: 10.9 days. The mean length of stay appeared to have stabilized at around 11.5 days in the years preceding 1989. The figure decreased even further to 10.5 days in 1990. In 1990, the occupancy rate in general hospitals also decreased by almost $2 \%$, from $69.1 \%$ (which is already quite low) in 1989 to $67.9 \%$ in 1990 . At the start of the eighties the occupancy rate in Dutch hospitals was still almost $80 \%$. Despite the unfortunate fact that we do not have access to recent procedure-specific length of stay data to enable us to examine the effects of the renewed budgeting system on the mean length of stay, we can still conclude on the basis of the above figures that the development in the mean length of stay in Dutch general hospitals (once again) shows a decreasing trend. Very recent medical developments, such as 'keyhole surgery' will reinforce this trend in the short term.

Could government regulation in the form of the development of a national system for standardization of the procedure-specific length of stay be an instrument for influencing the local length of stay standard? In the context of reducing the costs of treatment, a proposal in that direction was submitted in the Tweede Kamer, the lower house of the Dutch parliament (1988), to the then State Secretary for Health. The State Secretary subsequently asked the advice of the Ziekenfondsraad (national board for public health insurance). The Ziekenfondsraad subsequently brought out a negative advice, and stated, among a number of other things, that it is important to motivate health insurers to involve the aspect length of stay in the negotiations with hospital administrations (1989). In other words: deregulation. Besides the costs involved in a national system for standard- 
ization of the length of stay, it indeed seems more effective to tackle the differences in procedure-specific length of stay closer to the level of individual action. National indicators such as the mean procedurespecific length of stay, may serve hereby as a mirror.

Finally, we wish to mention one more instrument for influencing the differences in local standards of medical practice. This instrument already appeared to be reasonably successful in the United States. According to this method, the differences in local standards can be reduced by holding consensus conferences in which doctors from one certain medical specialty-from the entire country-exchange experiences and attempt to coordinate their medical activities, in this case the procedure-specific length of stay. In such an environment, local standards can be critically examined by colleagues and possibly adjusted in the direction of national standards.

Besides the fact that the policy developments in Dutch health care sketched above - the reduced importance of the patient day as budget parameter and the government's bed reduction policy-might have a favourable effect on the reduction of differences in local length of stay standards, the instrument of consensus conferences is a direct method which allows doctors to regulate their own activities and therefore to work on a further professionalization of the medical sector. However, we wish to emphasize that the effect of consensus conferences alone should not be overestimated.

Restrictions in doctors' work environment may discourage them from following the practice guidelines reached in the conference room. The following quote from Schroeder and Lo is illustrative in this respect:

(...) In chronic diseases such as peptic ulcer disease, an important part of the care is educating the patient and discussing how lifestyle, stress, diet, cigarettes and alcohol affect the disease. Yet the physician reimbursement schedule offers disincentives for such education and discussion. If Doctor $\mathbf{R}$ spends 25 minutes performing endoscopy, $s /$ he charges $\$ 300$, a fee that will be totally reimbursed by the patient's insurance. However, if $s /$ he spends 45 minutes in education and counselling, s/he charges $\$ 60$, which may not be covered by the patient's insurance [11].

\section{REFERENCES}

1. Chassin M. Variations in Hospital Length of Stay: Their Relationship to Health Outcomes. Office of Technology Assessment (OTA) Health Technology Case Study 24, Washington, 1983.

2. Copenhagen Collaborating Center for the Study of Regional Variations in Health Care. CCC Bibliography on Regional Variations in Health Care. Vedbak, 1985.

3. Gornick $\mathbf{M}$. Medicare patients: regional differences in length of hospital stays, 1969-1971. Soc. Security Bull. 38, $16-33,1975$.

4. West R. R. and Roberts C. J. Some observations on the management of appendicitis in Wales. Int. J. Epidemiol. 3, 351-357, 1974

5. West R. R. and Carey M. J. Variation in rates of hospital admission for appendicitis in Wales. Br. Med. J. 288, 1662-1664, 1978.

6. Sanders D., Coulter A. and McPherson K. Variations in Hospital Admission Rates: A Review of the Literature. Clinical Epidemiology Unit, University of Oxford, Oxford, 1988.

7. Wennberg J. E. and Gittelsohn A. Variations in medical care among small areas. Sci. Am. 246, 100-111, 1982.

8. Eddy D. M. Variations in physician practice: the role of uncertainty. Hlth Affairs 3, 74-89, 1984.

9. Griffiths M., Waters W. E. and Acheson E. D. Variation in hospital stay after inguinal hernirrhaphy. Br. Med.J. 289, 787-789, 1979.

10. Westert G. P. Variation in Use of Hospital Care. Books International, Herndon, VA, 1992.

11. Schroeder S. A. and Lo B. Reimbur sement incentives and clinical strategies. In The Machine at the Bedside (Edited by Reiser S. J. and Anbar M.). Cambridge University Press, Cambridge, MA, 1984.

\section{APPENDIX}

Numerical Data on Which the Figures 1 and 2 are Based

Figure 1

\begin{tabular}{cccc}
\hline & Minimum & Maximum & Average \\
\hline & \multicolumn{3}{c}{ Appendectomy } \\
A & 8.5 & 9.5 & 8.8 \\
B & 7.2 & 8.8 & 8 \\
C & 8.5 & 8.55 & 8.5 \\
D & 6.5 & 7.8 & 7.2 \\
E & 6.6 & 6.9 & 6.7 \\
& & Inguinal herniorraphy & \\
A & 5.7 & 6.7 & 6.1 \\
B & 6.2 & 7 & 6.5 \\
C & 6.3 & 7 & 6.6 \\
D & 3.8 & 7.1 & 5.6 \\
E & 5.4 & 6.4 & 6 \\
\hline
\end{tabular}

Figure 2

\begin{tabular}{rcc}
\hline$X$-axis & $Y$-axis & Database \\
\hline-0.7 & 0.5 & $\mathrm{~A}$ \\
-0.5 & 0.4 & $\mathrm{~A}$ \\
-0.4 & 0.5 & $\mathrm{~A}$ \\
0.0 & 0.3 & $\mathrm{~A}$ \\
3.9 & 3.3 & $\mathrm{~A}$ \\
7.2 & 0.3 & $\mathrm{~A}$ \\
1.3 & 2.2 & $\mathrm{~A}$ \\
1.0 & 1.4 & $\mathrm{~A}$ \\
-1.4 & 0.0 & $\mathrm{~A}$ \\
0.5 & 0.7 & $\mathrm{~A}$ \\
0.7 & 0.2 & $\mathrm{~A}$ \\
-3.6 & 0.7 & $\mathrm{~A}$ \\
0.8 & 0.7 & $\mathrm{~A}$ \\
2.1 & 1.3 & $\mathrm{~A}$ \\
-0.3 & 1.6 & $\mathrm{~A}$ \\
1.6 & 1.1 & $\mathrm{~A}$ \\
1.7 & 1.0 & $\mathrm{~A}$ \\
1.6 & 1.9 & $\mathrm{~A}$ \\
0.8 & 0.5 & $\mathrm{~A}$ \\
3.4 & 2.5 & $\mathrm{~A}$ \\
1.2 & 1.9 & $\mathrm{~B}$ \\
1.9 & 1.6 & $\mathrm{~B}$ \\
1.4 & 1.1 & $\mathrm{~B}$ \\
1.7 & 0.8 & $\mathrm{~B}$ \\
\hline
\end{tabular}

